\title{
Vaginal Assisted Delivery
}

National Cancer Institute

\section{Source}

National Cancer Institute. Vaginal Assisted Delivery. NCI Thesaurus. Code C81301.

Birth of the fetus through the vagina with the application of vacuum or forceps or any other instrument. 